\title{
Effects of Dietary Supplementation Rumen Protected Methionine, Lysine and Choline Supplementation on Feed Intake in Transition Murrah Buffaloes
}

\author{
Khwairakpam Ratika ${ }^{1 *}$ and R.K. James Singh ${ }^{2}$ \\ ${ }^{1}$ Division of Animal Nutrition, ICAR-National Dairy Research Institute, Karnal-132001, India \\ ${ }^{2}$ Division of Veterinary Biotechnology, ICAR-Indian Veterinary Research Institute, Izatnagar, \\ Bareilly-243122, India \\ *Corresponding author
}

A B S T R A C T

Keywords

Murrah Buffaloes, Lysine and choline supplementation, Intake

Article Info

Accepted:

07 June 2019

Available Online:

10 July 2019
A study was carried out to discern the effect of dietary incorporation of rumen protected methionine (RPM), lysine (RPL) and choline (RPC) on feed intake in transition Murrah buffaloes. Based on previous lactation milk yield and body weight, 20 buffaloes $\left(2^{\text {nd }}\right.$ to $4^{\text {th }}$ lactation $)$ in their third trimester were divided into four groups and fed as follows, Group I (control): basal diet consisting of wheat straw ad lib+ green fodder + concentrate mixture as per ICAR (2013) feeding standard; Group II: basal diet supplemented with $7 \mathrm{~g}$ RPM $+15 \mathrm{~g}$ RPL; Group III: basal diet supplemented with 50g RPC and Group IV: basal diet supplemented with $7 \mathrm{~g} \mathrm{RPM}+15 \mathrm{~g}$ RPL $+50 \mathrm{~g}$ RPC. The feeding trial was of 180 days duration (90 days before and 90 days postpartum). The DM intake was recorded at fortnightly interval for two consecutive days. And the results showed that there was no significant difference in total dry matter intake of buffaloes of all four groups both in pre-partum and post- partum period due to dietary supplementation of rumen protected methionine, lysine and choline.

\section{Introduction}

Transition period defined as last 3 weeks before parturition to first 3 weeks after parturition is often a disastrous experience for the buffaloes (Goff and Horst, 1997). This period is characterized by dramatic physiological and metabolic adaptations, and the animal is at the highest risk of succumbing to health disorder during this period (Loor et al., 2013). There is reduction in appetite and low intake of dry matter which is responsible for depressed performance specifically during the transition period. As the gestation advances, the dry matter intake (DMI) of dairy animals declines and DMI is at lowest (1.7- 
$2.0 \%$ of body weight) at parturition. So, proper nutritional management during this period regulates both the milk production in the proceeding lactation as well as the reproductive efficiency of the animal. Free methionine and lysine as the most limiting amino acids in dairy cows would be mostly degraded in the rumen and usually supplemented in the rumen-protected form.

Choline, a component of phospholipid and methyl donor, plays an essential role in very low density lipoprotein synthesis and thereby contributes to fat export from the liver. Supplementation of rumen protected methionine, lysine and choline has shown to improve milk production in dairy animals (Socha et al., 2005, Noftsger et al., 2005 and $\mathrm{Xu}$ et al., 2006). Effect on DMI due supplementation of rumen-protected methionine, lysine and choline in dairy animals had an inconsistent outcome. So, this study was conducted to see the effect of these rumen protected nutrients on DMI in transition Murrah buffaloes.

\section{Materials and Methods}

Twenty Murrah buffaloes of $2^{\text {nd }}$ to $4^{\text {th }}$ lactation, in $3^{\text {rd }}$ trimester of their pregnancy were divided into four groups having 5 animals in each group on the basis of their previous lactation yield and body weight. The concentrate mixture was offered twice daily i.e. half of the daily allowance at 9:00 AM and remaining half at 3:00 PM. Green fodder was offered at around 5:00 PM after the evening milking. All the animals had $a d$ lib access to wheat straw. The dry matter intake was recorded every fortnightly over two consecutive day and the animals were kept tied in the shed during these two days.

G I: (Basal diet) available green $20 \mathrm{~kg}+$ concentrate mixture as per ICAR (2013) feeding standard + wheat straw ad lib
$\mathrm{G}$ II: Basal diet $+7 \mathrm{~g}$ rumen protected methionine $+15 \mathrm{~g}$ rumen protected lysine

G III: Basal diet $+50 \mathrm{~g}$ rumen protected choline

G IV: Basal diet $+7 \mathrm{~g}$ rumen protected methionine $+15 \mathrm{~g}$ rumen protected lysine + $50 \mathrm{~g}$ rumen protected choline

The feeding trial was carried out for 6 months period during transition period of buffaloes i.e.

3 months before parturition to 3 months after parturition when animal transits from dry to lactation stage.

\section{Results and Discussion}

Dry matter intake (DMI) was recorded fortnightly for two consecutive days by recording feed offered and residues.

The dry matter of different feed ingredients was also estimated every 15 days.

\section{Pre-partum feed intake}

The results of pre-partum feed intake of Murrah buffaloes due to dietary incorporation of rumen protected methionine, lysine and choline are shown in Table 1.

From the results, it was observed that total DMI, DMI per $100 \mathrm{~kg}$

Body weight and DMI per metabolic body weight $\left(\mathrm{W}^{0.75}\right)$ were comparable in buffaloes of all four groups in all fornights.

\section{Post-partum feed intake}

The results of post-partum feed intake of Murrah buffaloes due to dietary incorporation of rumen protected methionine, lysine and choline are shown in Table 2. 
Table.1 Prepartum Average dry matter intake (DMI) of Murrah buffaloes supplemented with methionine, lysine and choline

\begin{tabular}{|c|c|c|c|c|c|c|}
\hline \multirow[t]{2}{*}{ Fornights } & \multicolumn{6}{|c|}{ Dry matter intake (g) } \\
\hline & G1 & \multirow[t]{2}{*}{ G2 } & \multirow[t]{2}{*}{ G3 } & G4 & \multirow[t]{2}{*}{ Mean \pm SEM } & \multirow{2}{*}{$\begin{array}{c}\mathrm{P}- \\
\text { value }\end{array}$} \\
\hline \multicolumn{3}{|l|}{ I $^{\text {st }}$ Fornight } & & & & \\
\hline Total DMI (kg) & $\begin{array}{l}12.63 \pm 1.0 \\
3\end{array}$ & $\begin{array}{l}12.30 \pm 0.5 \\
6\end{array}$ & $\begin{array}{l}12.83 \pm 0.3 \\
0\end{array}$ & $\begin{array}{l}12.58 \pm 1.1 \\
2\end{array}$ & $12.58 \pm 1.56$ & 0.796 \\
\hline DMI/100 kg BW(kg) & $2.27 \pm 0.20$ & $2.20 \pm 0.20$ & $2.34 \pm 0.09$ & $2.22 \pm 0.20$ & $2.25 \pm 0.34$ & 0.718 \\
\hline $\mathrm{DMI} / \mathrm{W}^{0.75} \mathrm{~kg}(\mathrm{~g})$ & $\begin{array}{l}19.92 \pm 1.1 \\
2\end{array}$ & $\begin{array}{l}19.35 \pm 2.8 \\
5\end{array}$ & $\begin{array}{l}20.45 \pm 1.1 \\
0\end{array}$ & $\begin{array}{l}19.07 \pm 1.9 \\
7\end{array}$ & $19.70 \pm 0.94$ & 0.764 \\
\hline \multicolumn{7}{|l|}{ II $^{\text {nd }}$ Fornight } \\
\hline Total DMI (kg) & $\begin{array}{l}12.73 \pm 1.1 \\
6\end{array}$ & $\begin{array}{l}12.68 \pm 1.7 \\
6\end{array}$ & $\begin{array}{l}13.68 \pm 0.8 \\
1\end{array}$ & $\begin{array}{l}12.47 \pm 1.3 \\
1\end{array}$ & $12.89 \pm 0.58$ & 0.484 \\
\hline DMI/100 kg BW(kg) & $2.26 \pm 0.25$ & $2.23 \pm 0.34$ & $2.44 \pm 0.15$ & $2.16 \pm 0.27$ & $2.27 \pm 0.12$ & 0.401 \\
\hline DMI/W ${ }^{0.75}$ kg (g) & $\begin{array}{l}19.63 \pm 3.0 \\
6\end{array}$ & $\begin{array}{l}19.31 \pm 3.4 \\
2\end{array}$ & $\begin{array}{l}21.13 \pm 1.3 \\
5\end{array}$ & $\begin{array}{l}18.39 \pm 2.7 \\
3\end{array}$ & $19.57 \pm 1.22$ & 0.472 \\
\hline \multicolumn{7}{|l|}{ III $^{\text {rd }}$ Fornight } \\
\hline Total DMI (kg) & $\begin{array}{l}12.90 \pm 1.1 \\
5\end{array}$ & $\begin{array}{l}12.59 \pm 1.2 \\
7\end{array}$ & $\begin{array}{l}12.89 \pm 0.7 \\
9\end{array}$ & $\begin{array}{l}12.60 \pm 0.8 \\
6\end{array}$ & $12.75 \pm 0.44$ & 0.935 \\
\hline DMI/100 kg BW(kg) & $2.26 \pm 0.26$ & $2.16 \pm 0.25$ & $2.26 \pm 0.16$ & $2.14 \pm 0.17$ & $2.21 \pm 0.10$ & 0.733 \\
\hline $\mathrm{DMI} / \mathrm{W}^{0.75} \mathrm{~kg}(\mathrm{~g})$ & $\begin{array}{l}19.37 \pm 3.1 \\
1\end{array}$ & $\begin{array}{l}18.26 \pm 2.8 \\
5\end{array}$ & $\begin{array}{l}19.39 \pm 1.5 \\
6\end{array}$ & $\begin{array}{l}17.97 \pm 1.6 \\
5\end{array}$ & $15.75 \pm 1.02$ & 0.701 \\
\hline \multicolumn{7}{|l|}{ IV $^{\text {th }}$ Fornight } \\
\hline Total DMI (kg) & $\begin{array}{l}12.94 \pm 1.0 \\
5\end{array}$ & $\begin{array}{l}12.43 \pm 1.5 \\
0\end{array}$ & $\begin{array}{l}13.18 \pm 0.8 \\
1\end{array}$ & $\begin{array}{l}12.52 \pm 0.6 \\
8\end{array}$ & $12.78 \pm 0.44$ & 0.653 \\
\hline DMI/100 kg BW(kg) & $2.21 \pm 0.13$ & $2.10 \pm 0.32$ & $2.25 \pm 0.16$ & $2.08 \pm 0.16$ & $2.16 \pm 0.40$ & 0.507 \\
\hline DMI/W $W^{0.75} \mathrm{~kg}(\mathrm{~g})$ & $\begin{array}{l}18.74 \pm 1.7 \\
5\end{array}$ & $\begin{array}{l}17.57 \pm 3.4 \\
6\end{array}$ & $\begin{array}{l}18.91 \pm 1.6 \\
1\end{array}$ & $\begin{array}{l}17.20 \pm 1.6 \\
8\end{array}$ & $18.10 \pm 0.98$ & 0.564 \\
\hline \multicolumn{7}{|l|}{$V^{\text {th }}$ Fornight } \\
\hline Total DMI (kg) & $\begin{array}{l}12.58 \pm 0.5 \\
6\end{array}$ & $\begin{array}{l}12.47 \pm 1.1 \\
1\end{array}$ & $\begin{array}{l}13.10 \pm 0.7 \\
0\end{array}$ & $\begin{array}{l}12.45 \pm 0.8 \\
1\end{array}$ & $12.65 \pm .34$ & 0.567 \\
\hline DMI/100 kg BW(kg) & $2.12 \pm 0.10$ & $2.07 \pm 0.24$ & $2.20 \pm 0.13$ & $2.03 \pm 0.18$ & $2.10 \pm 0.08$ & 0.516 \\
\hline DMI/W ${ }^{0.75}$ kg (g) & $\begin{array}{l}17.74 \pm 1.5 \\
0\end{array}$ & $\begin{array}{l}17.11 \pm 2.7 \\
4\end{array}$ & $\begin{array}{l}18.12 \pm 1.3 \\
3\end{array}$ & $\begin{array}{l}16.56 \pm 1.8 \\
2\end{array}$ & $17.38 \pm 0.82$ & 0.599 \\
\hline \multicolumn{7}{|l|}{ VI ${ }^{\text {th }}$ Fornight } \\
\hline Total DMI (kg) & $\begin{array}{l}12.54 \pm 0.5 \\
9\end{array}$ & $\begin{array}{l}12.19 \pm 1.3 \\
1\end{array}$ & $\begin{array}{l}12.95 \pm 0.5 \\
2\end{array}$ & $\begin{array}{l}12.53 \pm 0.5 \\
7\end{array}$ & $12.55 \pm 0.34$ & 0.548 \\
\hline DMI/100 kg BW(kg) & $2.08 \pm 0.19$ & $1.99 \pm 0.23$ & $2.14 \pm 0.09$ & $2.02 \pm 0.16$ & $2.06 \pm 0.06$ & 0.519 \\
\hline $\mathrm{DMI} / \mathrm{W}^{0.75} \mathrm{~kg}(\mathrm{~g})$ & $\begin{array}{l}17.22 \pm 1.6 \\
5\end{array}$ & $\begin{array}{l}16.32 \pm 2.6 \\
1\end{array}$ & $\begin{array}{l}17.54 \pm 0.8 \\
9\end{array}$ & $\begin{array}{l}16.30 \pm 1.6 \\
6\end{array}$ & $16.84 \pm 0.08$ & 0.621 \\
\hline
\end{tabular}

Value with different superscripts between columns differ significant $(\mathrm{P}<0.05)$. $(\mathrm{G} 1=$ Control group, $\mathrm{G} 2=\mathrm{RPM}+$ RPL supplemented group, G3= RPC supplemented group and G4= RPM +RPL+RPC supplemented group) 
Table.2 Postpartum Average dry matter intake (DMI) of Murrah buffaloes supplemented with methionine, lysine and choline

\begin{tabular}{|c|c|c|c|c|c|c|}
\hline \multirow[t]{2}{*}{ Fornights } & \multicolumn{6}{|c|}{ Dry Matter Intake (DMI) } \\
\hline & G1 & G2 & G3 & G4 & Mean \pm SEM & $\begin{array}{l}\mathrm{p}- \\
\text { value }\end{array}$ \\
\hline \multicolumn{7}{|l|}{$\mathrm{I}^{\mathrm{st}}$ Fornight } \\
\hline Total DMI (kg) & $\begin{array}{l}12.28 \pm 1.3 \\
9\end{array}$ & $\begin{array}{l}13.29 \pm 1.9 \\
3\end{array}$ & $\begin{array}{l}13.44 \pm 0.6 \\
7\end{array}$ & $\begin{array}{l}13.19 \pm 0.9 \\
5\end{array}$ & $13.05 \pm 0.48$ & 0.338 \\
\hline DMI/100 kg BW(kg) & $2.32 \pm 0.26$ & $2.45 \pm 0.21$ & $2.51 \pm 0.11$ & $2.38 \pm 0.12$ & $2.41 \pm 0.36$ & 0.427 \\
\hline $\mathrm{DMI} / \mathrm{W}^{0.75} \mathrm{~kg}(\mathrm{~g})$ & $\begin{array}{l}21.04 \pm 3.0 \\
3\end{array}$ & $\begin{array}{l}21.87 \pm 2.8 \\
1\end{array}$ & $\begin{array}{l}22.48 \pm 1.2 \\
1\end{array}$ & $\begin{array}{l}20.84 \pm 1.1 \\
1\end{array}$ & $21.55 \pm 4.2$ & 0.632 \\
\hline \multicolumn{7}{|l|}{ II $^{\text {nd }}$ Fornight } \\
\hline Total DMI (kg) & $\begin{array}{l}12.00 \pm 1.2 \\
6\end{array}$ & $\begin{array}{l}12.66 \pm 1.4 \\
5\end{array}$ & $\begin{array}{l}13.13 \pm 0.7 \\
7\end{array}$ & $\begin{array}{l}12.52 \pm 0.5 \\
9\end{array}$ & $12.58 \pm 0.44$ & 0.373 \\
\hline DMI/100 kg BW(kg) & $2.37 \pm 0.29$ & $2.44 \pm 0.25$ & $2.56 \pm 0.12$ & $2.34 \pm 0.09$ & $2.42 \pm 0.08$ & 0.388 \\
\hline DMI/W ${ }^{0.75} \mathrm{~kg}(\mathrm{~g})$ & $\begin{array}{l}22.32 \pm 4.0 \\
7\end{array}$ & $\begin{array}{l}22.58 \pm 3.4 \\
7\end{array}$ & $\begin{array}{l}23.69 \pm 1.5 \\
6\end{array}$ & $\begin{array}{l}21.02 \pm 1.1 \\
3\end{array}$ & $22.40 \pm 1.2$ & 0.542 \\
\hline \multicolumn{7}{|l|}{ III $^{\text {rd }}$ Fornight } \\
\hline Total DMI (kg) & $\begin{array}{l}11.84 \pm 1.0 \\
2\end{array}$ & $\begin{array}{l}12.73 \pm 1.2 \\
7\end{array}$ & $\begin{array}{l}12.35 \pm 0.7 \\
0\end{array}$ & $\begin{array}{l}12.34 \pm 1.2 \\
7\end{array}$ & $12.32 \pm 0.48$ & 0.653 \\
\hline DMI/100 kg BW(kg) & $2.36 \pm 0.16$ & $2.48 \pm 0.29$ & $2.38 \pm 0.16$ & $2.30 \pm 0.17$ & $2.38 \pm 0.8$ & 0.588 \\
\hline $\mathrm{DMI} / \mathrm{W}^{0.75} \mathrm{~kg}(\mathrm{~g})$ & $\begin{array}{l}22.33 \pm 2.8 \\
3\end{array}$ & $\begin{array}{l}23.08 \pm 3.7 \\
9\end{array}$ & $\begin{array}{l}21.97 \pm 1.7 \\
1\end{array}$ & $\begin{array}{l}20.72 \pm 1.2 \\
9\end{array}$ & $22.02 \pm 1.12$ & 0.555 \\
\hline \multicolumn{7}{|l|}{ IV $^{\text {th }}$ Fornight } \\
\hline Total DMI (kg) & $\begin{array}{l}11.80 \pm 1.1 \\
9\end{array}$ & $\begin{array}{l}12.70 \pm 1.7 \\
9\end{array}$ & $\begin{array}{l}12.66 \pm 1.1 \\
2\end{array}$ & $\begin{array}{l}12.68 \pm 0.7 \\
4\end{array}$ & $12.46 \pm 0.54$ & 0.630 \\
\hline DMI/100 kg BW(kg) & $2.34 \pm 0.18$ & $2.49 \pm 0.37$ & $2.45 \pm 0.20$ & $2.35 \pm 0.11$ & $2.41 \pm 0.10$ & 0.666 \\
\hline $\mathrm{DMI} / \mathrm{W}^{0.75} \mathrm{~kg}(\mathrm{~g})$ & $\begin{array}{l}22.06 \pm 2.8 \\
9\end{array}$ & $\begin{array}{l}23.38 \pm 4.3 \\
5\end{array}$ & $\begin{array}{l}22.72 \pm 1.9 \\
1\end{array}$ & $\begin{array}{l}20.98 \pm 1.3 \\
1\end{array}$ & $22.28 \pm 1.24$ & 0.601 \\
\hline \multicolumn{7}{|l|}{$\mathrm{V}^{\text {th }}$ Fornight } \\
\hline Total DMI (kg) & $\begin{array}{l}11.87 \pm 1.1 \\
4\end{array}$ & $\begin{array}{l}12.81 \pm 1.0 \\
3\end{array}$ & $\begin{array}{l}12.68 \pm 0.3 \\
7\end{array}$ & $\begin{array}{l}12.63 \pm 1.4 \\
2\end{array}$ & $12.50 \pm 0.46$ & 0.513 \\
\hline DMI/100 kg BW(kg) & $2.35 \pm 0.27$ & $2.51 \pm 0.27$ & $2.47 \pm .08$ & $2.35 \pm 0.21$ & $2.42 \pm 0.10$ & 0.593 \\
\hline $\mathrm{DMI} / \mathrm{W}^{0.75} \mathrm{~kg}(\mathrm{~g})$ & $\begin{array}{l}22.32 \pm 3.9 \\
0\end{array}$ & $\begin{array}{l}23.47 \pm 3.7 \\
5\end{array}$ & $\begin{array}{l}23.02 \pm 1.0 \\
6\end{array}$ & $\begin{array}{l}21.19 \pm 1.9 \\
6\end{array}$ & $22.50 \pm 1.26$ & 0.638 \\
\hline \multicolumn{7}{|l|}{$\mathrm{VI}^{\text {th }}$ Fornight } \\
\hline Total DMI (kg) & $\begin{array}{l}12.31 \pm 0.7 \\
0\end{array}$ & $\begin{array}{l}13.03 \pm 1.3 \\
1\end{array}$ & $\begin{array}{l}12.98 \pm 0.4 \\
2\end{array}$ & $\begin{array}{l}12.89 \pm 0.9 \\
4\end{array}$ & $12.80 \pm 0.38$ & 0.576 \\
\hline DMI/100 kg BW(kg) & $2.45 \pm 0.27$ & $2.52 \pm 0.33$ & $2.54 \pm 0.12$ & $2.40 \pm 0.13$ & $2.48 \pm 0.10$ & 0.754 \\
\hline $\mathrm{DMI} / \mathrm{W}^{0.75} \mathrm{~kg}(\mathrm{~g})$ & $\begin{array}{l}23.15 \pm 4.3 \\
6\end{array}$ & $\begin{array}{l}23.48 \pm 4.1 \\
8\end{array}$ & $\begin{array}{l}23.70 \pm 1.6 \\
1\end{array}$ & $\begin{array}{l}21.54 \pm 1.6 \\
3\end{array}$ & $22.96 \pm 1.38$ & 0.715 \\
\hline
\end{tabular}

Value with different superscripts between columns differ significant $(\mathrm{P}<0.05)$. $(\mathrm{G} 1=$ Control group, $\mathrm{G} 2=\mathrm{RPM}+$ RPL supplemented group, G3= RPC supplemented group and G4= RPM +RPL+RPC supplemented group) 
From the results, it was observed that in all fornights during postpartal period, there was no effect of supplementation of rumen protected methionine, lysine and choline on total DMI, DMI per $100 \mathrm{~kg}$ body weight and DMI per metabolic body weight $\left(\mathrm{W}^{0.75}\right)$ of buffaloes of all treatment groups.

The present study indicated that dietary supplementation of rumen protected methionine, lysine and choline had no effect on DMI of buffaloes and are in agreement with the study conducted by Donkin et al., (1989); Armentano et al., (1993) and Chung (2003) who also reported that rumen protected methionine and lysine had no effect on DMI in Holstein dairy cows. Similarly no effect on DMI was reported by Ahmed et al., (2016) in Nili-ravi buffaloes on supplementation of rumen protected lysine and methionine. Lima et al., (2007) and Leiva et al., (2015) also noticed no effect on DMI due to RPC supplementation in transition cows. Elek et al., (2008); Suksombat et al., (2012); Guretzky et al., (2006) and Rahmani et al., (2014) showed no effect on DMI in choline supplemented group in Holstein cows.

Contrary to our findings, Ordway et al., (2009) observed greater postpartum intake in rumen protected methionine supplemented group in Holstein Friesian cows. Soltan et al., (2012) also reported improved DMI in RPM and RPC supplemented groups of Holstein Friesian cows. Sheikh et al., (2014) also indicated that supplementation of rumen protected methionine, lysine and choline in Karan Fries cows improved dry matter intake.

\section{References}

Ahmed, S., Gohar, M., Khalique, A., Ahmad, N., Shahzad, F., Burhan-e-Azam, Rahman, A. and Khan, M. I. (2016). Effect of Supplementation of Rumen Protected Lysine and Methionine on
Production Performance, Milk and Blood Parameters of Early Lactating Nili-Ravi Buffaloes. Pakistan Journal of Zoology, 48(2): 359-363.

Armentano, L. E. and Swain, S. M. (1993). Lactation response to ruminally protected methionine and lysine at two amounts of ruminally available nitrogen. Journal of Dairy Science, 76: 2963-2969.

Chung, Y. H. (2003). Effects of free methionine and lysine on in vitro fermentation and in vivo performance performance and ruminal fermentation of late lactation Holstein cows. Master's thesis. B. S., Chinese Culture University, Taiwan.

Donkin, S. S., Varga, G. A., Sweeney, T. F. and Muller, L. D. (1989). Rumenprotected methionine and lysine: effects on animal performance, milk protein yield and physiological measures. Journal of Dairy Science, 72: 14841491.

Elek, P., Newbold, J. R., Gall, T., Wagner, L. and Husventh, F. (2008). Effects of rumen protected choline supplementation on milk production and choline supply of peri-parturient dairy cows. Animal, 2(11):1595-1601.

Goff, J. P. and Horst, R. L. (1997). Physiological changes at parturition and their relationship to metabolic disorders. Journal of Dairy Science, 80(7):1260-8.

Guretzky, N. A. J., Carlson, D. B., Garrett, J. E. and Drackley, J. K. (2006). Lipid Metabolite Profiles and Milk Production for Holstein and Jersey Cows Fed Rumen-Protected Choline during the Periparturient Period. Journal of Dairy Science, 89: 188-200.

Leiva, T., Cooke, R. F., Brandao, A. P., Marques, R. S. and Vasconcelos, J. L. (2015). Effect of rumen-protected choline supplementation on metabolic and performance responses of transition 
dairy cows. Journal of Dairy Science, 93(4):1896-1904.

Lima, F. S., Sa Filho, M. F., Greco, L. F., Susca, F., Magalhaes, V. J., Garrett, J. and Santos, J. P. E. (2007). Effects of feeding rumen-protected choline (RPC) on lactation and metabolism. Journal of Dairy Sciences, 90(1):174.

Loor, J. J., Bionaz, M. and Drackley, J. K. (2013). Systems physiology in dairy cattle: Nutritional genomics and beyond. Annual Review of Animal Biosciences, 1: 365-392.

Noftsger, S., St-Pierre, N. R. and Sylvester, J. T. (2005). Determination of rumen degradability and ruminal effects of three sources of methionine in lactating cows. Journal of Dairy Science, 88: 223-237.

Ordway, R. S., Boucher, S. E., Whitehouse, N. L., Schwab, C. G. and Sloan, B. K. (2009). Effects of providing two forms of supplemental methionine to periparturient Holstein dairy cows on feed intake and lactational performance. Journal of Dairy Science, 92(10): 51545166.

Rahmani, M., Dehghan- Banadaky, M. and Kamalyan, R. (2014). Effects of Feeding Rumen Protected Choline and Vitamin E on Milk Yield, Milk Composition, Dry Matter Intake, Body Condition Score and Body Weight in Early Lactating Dairy Cows. Iranian Journal of Applied Animal Science, 4(4): 693-698.

Sheikh, F. A., Kewalramani, N., Thakur, S. S. and Mir, I. A. (2014). Effect of
Supplementation of Rumen Protected Methionine-lysine and Choline on Milk Production in Crossbred Cows. Indian Journal of Animal Nutrition, 31 (2): 148-151.

Socha, M. T., Putnam, D. E., Garthwaite, B. D., Whitehouse, N. L., Kierstead, N. A., Schwab, C. G., Ducharme, G. A. and Robert, J. C. (2005). Improved intestinal amino acid supply of pre- and postpartum dairy cows with rumen protected methionine and lysine. Journal of Dairy Science, 88: 11131126.

Soltan, M. A., Mujalli, A. M., Mandour, M. A. and Abeer, M. E. (2012). Effect of dietary rumen protected methionine and/or choline supplementation of rumen fermentation characteristics and protective performance of early lactating cows. Pakistan Journal of Nutrition, 11(3): 221-230.

Suksombat, W., Homkao, J. and Klangnork, P. (2012). Effect of biotin and rumen protected choline supplementation on milk production, milk composition, live weight change and blood parameters in lactating dairy weights. Journal of Animal and Veterinary Advances, 10: 2186-2192.

Xu, G. Z., Ye, J. A., Liu, J. X. and Wu, Y. M. (2006). Effect of rumen-protected choline addition on milk performance and blood metabolic parameters in transition dairy cows. AsianAustralasian Journal of Animal Science, 19: 390-395.

\section{How to cite this article:}

Khwairakpam Ratika and James Singh, R.K. 2019. Effects of Dietary Supplementation Rumen Protected Methionine, Lysine and Choline Supplementation on Feed Intake in Transition Murrah Buffaloes. Int.J.Curr.Microbiol.App.Sci. 8(07): 779-784. doi: https://doi.org/10.20546/ijcmas.2019.807.094 